\title{
Criminologie
}

\section{Grandeurs et misères de la politique pénale au Canada : du réformisme au populisme}

\section{Pierre Landreville}

Volume 40, numéro 2, automne 2007

Peines et pénalité au Canada. Autour des travaux de Pierre Landreville

URI : https://id.erudit.org/iderudit/016850ar

DOI : https://doi.org/10.7202/016850ar

Aller au sommaire du numéro

Éditeur(s)

Les Presses de l'Université de Montréal

ISSN

0316-0041 (imprimé)

1492-1367 (numérique)

Découvrir la revue

Citer cet article

Landreville, P. (2007). Grandeurs et misères de la politique pénale au Canada : du réformisme au populisme. Criminologie, 40(2), 19-51.

https://doi.org/10.7202/016850ar
Résumé de l'article

Depuis quelques décennies, les observateurs constatent un durcissement très marqué des politiques et des pratiques pénales, surtout aux États-Unis. Plusieurs, dont David Garland et Loïc Wacquant, ont tenté de documenter et d'expliquer ce virage punitif. Cette thèse de la " globalisation » du virage punitif a suscité nombre de réactions et de questionnements et a été remise en question dans le cas du Canada. Malgré quelques efforts pour documenter empiriquement ces transformations du pénal, plusieurs discours théoriques sur les transformations du pénal, sur les effets de la globalisation et de la mondialisation partent d'une analyse trop sommaire et trop superficielle des politiques et des pratiques pénales. C'est pourquoi on met l'accent dans cet article sur la description de ce qui s'est passé au Canada au cours du dernier demi-siècle avant de tenter d'en dégager les grandes tendances et d'esquisser quelques hypothèses d'explication.
Ce document est protégé par la loi sur le droit d'auteur. L'utilisation des services d’Érudit (y compris la reproduction) est assujettie à sa politique d'utilisation que vous pouvez consulter en ligne.

https://apropos.erudit.org/fr/usagers/politique-dutilisation/ 


\title{
Grandeurs et misères de la politique pénale au Canada: du réformisme au populisme
}

\author{
Pierre Landreville \\ Professeur émérite \\ École de criminologie, Université de Montréal \\ pierre.landreville@umontreal.ca
}

RÉSUMÉ - Depuis quelques décennies, les observateurs constatent un durcissement très marqué des politiques et des pratiques pénales, surtout aux États-Unis. Plusieurs, dont David Garland et Loïc Wacquant, ont tenté de documenter et d'expliquer ce virage punitif. Cette thèse de la «globalisation» du virage punitif a suscité nombre de réactions et de questionnements et a été remise en question dans le cas du Canada. Malgré quelques efforts pour documenter empiriquement ces transformations du pénal, plusieurs discours théoriques sur les transformations du pénal, sur les effets de la globalisation et de la mondialisation partent d'une analyse trop sommaire et trop superficielle des politiques et des pratiques pénales. C'est pourquoi on met l'accent dans cet article sur la description de ce qui s'est passé au Canada au cours du dernier demi-siècle avant de tenter d'en dégager les grandes tendances et d'esquisser quelques hypothèses d'explication.

ABSTRACT - During the last few decades, observers have noticed a strong toughening of penal policies and practices, especially in the USA. Many, among them David Garland and Lö̈ Wacquant, have tried to document and explain this punitive turn. This thesis of the "globalisation" of that punitive turn has given rise to a number of reactions and interrogations and has been put into question in Canada. In spite of some efforts to empirically document these modifications, many theoretical discourses on the effects of globalisation are based on too superficial and succinct analysis. That is why, in this article, we emphasize what has happened in this field in Canada in the last half-century, before attempting to highlight the major trends and attempt to outline explanatory hypothesis. 


\section{Introduction}

Au terme d'une carrière, et particulièrement à l'occasion d'une publication comme celle-ci, on est irrésistiblement conduit à faire des bilans. On peut faire le point sur son propre cheminement et faire un bilan de ce qu'on a produit tant du point de vue quantitatif que qualitatif. Ce genre de réflexion est une démarche personnelle. On peut aussi tenter d'évaluer l'impact de nos travaux et se demander quelle a été notre contribution à l'avancement de la connaissance ou des politiques et des pratiques dans notre domaine. La tâche du criminologue en particulier n'est pas uniquement de comprendre la réalité, de tenter de mieux la connaître mais aussi celle de la changer, d'améliorer le champ de la justice pénale. Le criminologue, tel que je le conçois, est aussi un ingénieur social qui doit tenter de «changer le monde», le rendre plus juste, plus humain et d'améliorer le fonctionnement des institutions. Je me suis intéressé aux politiques et aux pratiques pénales et à ce sujet on peut se proposer de cerner les changements, les transformations de ces politiques et de ces pratiques qui se sont produites durant les dernières décennies, et essayer de comprendre ces transformations. C'est ce que je vais tenter de réaliser dans ce court essai.

J'ai entrepris mes études universitaires au début des années $1960 \mathrm{au}$ moment où s'amorçait la révolution tranquille, la transformation rapide du Québec. Étudiant en sociologie, j'étais particulièrement sensible à ces transformations du système de l'éducation ou de la santé auxquelles participaient de plain-pied certains de mes professeurs ou de mes confrères plus âgés. Je voulais faire ma part et je me dirigeai en criminologie pour contribuer à l'humanisation, la modernisation du système de l'administration de la justice qui en avait grandement besoin.

Pour illustrer plus concrètement l'état du système pénal à cette époque et les besoins de changement, je ferai appel à quelques expériences personnelles. En 1963, lors d'un travail d'été au pénitencier de Saint-Vincent-de-Paul à titre d'«officier de classement» (préposé à l'évaluation et à la relation d'aide auprès des personnes incarcérées), j'ai rencontré un jeune de 19 ans qui avait tenté de se suicider. Il venait de recevoir un certain nombre de coups de fouet «chat à neuf queues» imposés comme peine par le tribunal après avoir été reconnu coupable de viol. Il redoutait tellement la seconde séance de flagellation, qui devait lui être infligée quelque mois plus tard, qu'il avait tenté de s'enlever la vie. Les châtiments corporels ne seront rayés du Code criminel 
qu'en 1969. Je me rappelle par ailleurs que mon premier projet de mémoire, qui devait s'avérer trop difficile à réaliser, portait sur l'impact de la présence ou de l'absence d'un avocat auprès d'un accusé lors de procès sur les décisions du tribunal. Un grand nombre d'accusés se présentaient en cour sans avocat, puisque le service public d'aide juridique ne fut créé qu'en 1972. Enfin, le premier texte que j'ai publié en 1965 dans le Quartier Latin, journal des étudiants de l'Université de Montréal, plaidait en faveur de la création d'un service de probation au Québec, service qui fut créé en 1967.

Ces quelques exemples permettent d'apprécier que le système pénal a changé depuis les années 1950-1960. Les châtiments corporels et la peine de mort ont été abolis, la plupart des accusés sont assistés d'un avocat, les peines non carcérales sont utilisées couramment et la Charte des droits de 1982 est venue consacrer et renforcer les droits et libertés des citoyens aux prises avec la justice. Mais, depuis quelques décennies, les observateurs constatent un durcissement très marqué des politiques et des pratiques pénales, surtout aux États-Unis.

Dans une communication au congrès de l'American Society of Crimino$\log y$ tenu à Miami en $1994^{1}$, David Garland notait que la plupart des analystes s'entendaient sur le fait que la pénalité subissait de profondes transformations, mais que, par ailleurs, il y avait peu de consensus sur la nature et les causes de ces transformations. Quelques années plus tard, ce même auteur (Garland, 2001) faisait une analyse du virage punitif, en se basant sur les cas des États-Unis et de la Grande-Bretagne, et expliquait que la nouvelle réponse sociale à la criminalité s'explique par des changements culturels et sociaux reliés à la modernité avancée et aux forces du marché néolibéral qui se sont développées durant les dernières décennies. Il laissait cependant entendre que sa thèse s'appliquait aux sociétés contemporaines.

Il n'est cependant pas le seul à mettre l'accent sur ce virage punitif et à tenter de l'expliquer. Déjà par exemple, au début des années 1990, Feeley et Simon (1992) ont attiré l'attention sur certaines tendances qu'ils croyaient voir se développer dans le domaine correctionnel. Dans cette «nouvelle pénologie», l'individualisation de la peine et les préoccupations pour la resocialisation devenaient fortement concurrencées

1. Garland (1995). Penal Modernism and Postmodernism. In T. G. Blomberg \& S. Cohen (eds.), Punishment and Social Control (181-210). New York: Aldine de Gruyer. 
par la gestion actuarielle des groupes, basée sur les probabilités et la notion de gestion du risque ${ }^{2}$.

Pour Wacquant (1999), l'ascension de l'État pénal, spectaculaire aux États-Unis, et cette expansion du «traitement pénal de la misère» découleraient de l'amputation de la capacité d'intervention sociale de l'État et viseraient à juguler les effets de l'insécurité sociale. D'une façon plus générale, un nouveau sens commun pénal néolibéral, venu d'Amérique, se propagerait en Europe ${ }^{3}$. Plus largement, Wacquant évoquait la tentation de s'appuyer sur les institutions judiciaire et pénitentiaire pour juguler les effets de l'insécurité sociale engendrés par l'imposition du salariat précaire et par le rétrécissement corrélatif de la protection sociale.

Cette thèse de la "globalisation» du virage punitif, particulièrement celle de Garland (2001), a suscité nombre de réactions et de questionnements ${ }^{4}$ et a été remise en question dans le cas du Canada. O'Malley (2006) fait une analyse de la situation canadienne et soutient quau Canada et en Australie «les tendances repérées aux États-Unis et en Angleterre sont assourdies et complexifiées car le système pénal y a conservé de robustes théories et pratiques de l'État-providence...» (p. 323). Dans un autre article (Meyer et O’Malley, 2005), les auteurs concluent de façon assez péremptoire: "Canadian criminal justice cannot be subsumed under a general model of global punitive turn. As has become clear in this analysis, policies and official discourses are turning as much and perhaps more to the forms of intervention characteristic of penal modernism» (p. 213). Moore et Hannah-Moffat (2005) s'intéressent particulièrement aux programmes dans les pénitenciers canadiens et concluent: «Punishment in Canada operates under different practices and policies that those suggested through the punitive turn thesis» (p. 97). Selon ces auteurs, le système pénitentiaire est encore orienté vers le changement des contrevenants où ceux-ci doivent se «responsabiliser» et sont incités à participer à leur propre changement. Il s'agit, soutiennent-ils, d'une forme différente de punition dans laquelle ce sont l'âme et les comportements des condamnés qui sont l'objet de la punition.

Ces tentatives de remise en question de la thèse du virage punitif sont intéressantes et louables dans le sens qu'elles documentent de façon

2. Voir aussi à ce sujet: Vacheret, Dozois et Lemire (1998) pour le Canada, et Mary (2001) pour l'Europe.

3. Pour une position plus nuancée à ce sujet voir Landreville (2002).

4. Voir entre autres Zedner (2002) et Pratt, Brown et al. (2005). 
empirique certaines des transformations du discours et des pratiques pénales. Nous estimons, en effet, que plusieurs discours théoriques sur les transformations du pénal, sur les effets de la globalisation et de la mondialisation partent d'une analyse trop sommaire et trop superficielle des politiques et des pratiques pénales. C'est pourquoi je mettrai l'accent sur la description de ce qui s'est passé au Canada avant de tenter d'en dégager les grandes tendances.

D’entrée de jeu, je soumets que les États-Unis sont un cas atypique parmi les pays occidentaux, avec un taux de détention qui s'est multiplié par quatre depuis 1970 pour atteindre 750 personnes incarcérées par 100000 habitants en 2006. Il faut se méfier de la tentation de croire que cette fureur répressive peu commune est un modèle inéluctable. Je crois aussi, à l'instar de plusieurs, que les transformations de la pénalité, particulièrement au Canada, sont plus complexes et moins globales que ne le prétend Garland. Elles ne sont pas univoques mais parfois ambiguës puisqu'elles sont le produit de tensions et de contradictions entre les divers groupes sociaux. Enfin, je fais l'hypothèse, à la suite de O’Malley (2006) et de Moore et Hannah-Moffat (2005), que malgré les discours politiques et scientifiques des dernières années, et même malgré certains courants législatifs, la résistance d'acteurs (procureurs, juges, administrateurs, intervenants des milieux correctionnels) qui partagent des valeurs de justice sociale, d'équité, de solidarité ou qui croient à la réhabilitation, fait en sorte que les pratiques pénales sont moins punitives que les discours et qu'il y a parfois un écart très considérable entre discours et pratique.

Je me propose donc d'esquisser la transformation du domaine de la détermination de la peine et de l'application des peines au Canada et au Québec au cours du dernier demi-siècle en distinguant, dans la mesure du possible, trois niveaux de réalité: le discours, la législation et les pratiques au sein du système pénal. Par discours, j'entends les discours savants (les analyses et les propositions faites par des experts universitaires ou par des membres de commissions), les rapports de comités ou de commissions, les documents d'énoncés de politiques, les déclarations d'intention et les propositions faites par des politiciens. Je veux aussi distinguer les lois, les règlements et les directives qui définissent ce qui doit être, et leur application. Nous savons tous, en effet, que certaines lois sont peu ou pas appliquées, que certaines sont formulées de façon à être presque inapplicables. Par pratiques, on doit entendre la façon dont les lois sont mises en œuvre, mais aussi les conséquences des 
politiques gouvernementales ou des décisions des administrateurs du système pénal.

Si le Code criminel, les pénitenciers et la Commission nationale des libérations conditionnelles sont du ressort du gouvernement fédéral, l'application de la loi est généralement une juridiction provinciale, ce qui fait en sorte que le Canada n'est pas un pays homogène du point de vue des pratiques pénales. D'autres facteurs, tels les taux de criminalité, très différents entre certaines provinces, et la répartition très différente des populations autochtones, accentuent l'hétérogénéité de ces pratiques. Mon esquisse ne pourra pas malheureusement tenir compte de toutes ces différences et manquera de nuances, selon certains. Je mettrai aussi évidemment l'accent, surtout lorsqu'il sera question des pratiques, sur la situation québécoise.

Mon texte sera divisé en six parties: 1) les transformations du discours de politique pénale; 2) les transformations législatives; 3) les transformations des pratiques pénales; 4) les grandes tendances et les paradoxes de la répression pénale; 5) quelques réflexions et hypothèses pour comprendre ces transformations; et finalement 6) une conclusion.

\section{Les transformations du discours de politique pénale}

\section{Les années 1950 à 1980}

Au Canada, les trois décennies 1950, 1960 et 1970 ont été marquées par des rapports importants visant la réforme $e^{5}$ des lois et du système pénal ${ }^{6}$. Retenons en particulier ceux de la Commission royale pour la révision du Code criminel (rapport Martin, 1954), du comité institué pour faire enquête sur les principes et méthodes suivis au Service des pardons du ministère de la Justice du Canada (rapport Fauteux, 1956), du Comité canadien de la réforme pénale et correctionnelle (rapport Ouimet, 1969), et des nombreux rapports et documents de travail de la Commission de réforme du droit du Canada instituée en 1970.

5. Cette période a été une période réformiste où le discours mettait l'accent sur la réforme, l'humanisation et la modernisation du droit et du système pénal, mais toujours à l'intérieur de la logique et du paradigme pénal. Il n'a jamais été question de remettre en cause «la rationalité pénale moderne» (Pires, 1998).

6. Pour une description plus détaillée, voir Laberge et Landreville (1991). 
Dans les années 1950, la Commission pour la révision du Code criminel avait pour objectif de «rafraîchir» le code, d'en éliminer les répétitions et d'en simplifier la présentation. Le Comité Fauteux, quant à lui, proposa une réforme en profondeur de la libération conditionnelle pour qu'elle constitue «une étape logique dans la réforme et la réadaptation d'une personne qui a été trouvée coupable d'un délit $[\ldots]$ » (p. 54).

En 1969, le Comité Ouimet prônait la modération dans l'usage du droit pénal et affirmait «que la réadaptation définitive de l'individu offre à la société sa meilleure protection à long terme [...]» (p. 204). Il préconisait, entre autres, une libéralisation de la mise en liberté sous caution, l'abolition de la peine corporelle, une diminution de l'usage de l'emprisonnement, une plus grande application de la probation, la création de la surveillance obligatoire pour soumettre les personnes libérées de façon statutaire aux deux tiers de leur peine aux mêmes conditions que les libérés conditionnels, et la création d'une forme de pardon pour atténuer les effets du casier judiciaire.

Les nombreux documents de la Commission de réforme du droit du Canada ont suscité des réflexions et alimenté les débats au sujet du droit pénal et des peines durant plus d'une décennie. Le leitmotiv de la Commission dans ce domaine était certes le principe de modération. Ses propositions de n'utiliser l'emprisonnement qu'en dernier ressort, de favoriser la déjudiciarisation et des mesures non carcérales telles le dédommagement, les travaux communautaires, l'amende, ont pris une place centrale dans les discours au sujet des politiques pénales au Canada. La Commission a été abolie à la fin des années 1980.

Au Québec, la Commission Prévost (1968), créée au début de 1967, a énoncé les grands principes qui allaient transfigurer la justice québécoise au cours de la décennie. Dès les premières pages de leur rapport, les commissaires formulent les exigences fondamentales de l'administration de la justice: «Profond respect des droits fondamentaux de la personne humaine, efficacité de la lutte au crime, harmonisation des divers éléments» (p. 16). On énonce ensuite certains buts particuliers dont une justice égale pour tous et le respect de la personne. Dans la perspective d'une justice égale pour tous, on signale trois problèmes: les peines pécuniaires, la détention due à la pauvreté et le besoin d'une assistance légale pour faciliter l'accès aux services d'un avocat. Quant au respect de la personne, on propose la réduction de l'usage de l'emprisonnement, jugé coûteux, inefficace et entraînant «une profonde 
dégradation de la personne humaine» (p. 51). On dénonce, dans la même veine, le recours abusif à la détention provisoire et on affirme le droit à la liberté avant le verdict ou l'imposition de la peine. Puis, soulignant que «notre administration de la justice devra également reconnaitre ses responsabilités à l'égard des personnes reconnues coupables» (p. 52), on affirme le droit au traitement adéquat pour les personnes incarcérées. Le volume 2 , intitulé «La sécurité judiciaire», analyse l'inégalité des justiciables devant la loi et suggère la création de services d'aide juridique accessibles à tous.

Le mouvement pour la défense et la reconnaissance des droits des personnes incarcérées s'est mis en branle au milieu des années 1970: un nouveau discours, fondé non pas sur l'amélioration des conditions de détention, mais sur les droits des détenus comme citoyens, s'est articulé de façon plus précise au cours de ces années (Landreville et al., 1976; Lemonde et Landreville, 2002). C'est aussi le moment où est entreprise l'action judiciaire proprement dite. Le mouvement a connu une croissance très rapide et en moins de dix ans, soit de 1975 à 1985, les principales bases des revendications juridiques étaient jetées.

\section{Depuis les années 1980 jusqu'à aujourd'hui}

On peut probablement considérer que les orientations des politiques pénales au Canada ont pris un certain tournant au milieu des années 1980. La période précédente de réforme se termine symboliquement par la venue au pouvoir des conservateurs en 1984. En 1987, le revirement se concrétisa par le dépôt d'un projet de loi ouvrant le débat sur le retour de la peine de mort, heureusement défait, et par la création sur la scène fédérale du Reform Party ${ }^{7}$ dont la plate-forme électorale est centrée sur des politiques pénales répressives.

Créée en 1984, la Commission canadienne sur la détermination de la peine (Archambault, 1987) a été saisie de la question de la disparité des sentences et de l'étude des lignes directrices en matière de détermination de la peine. Son rapport, Réformer la sentence: une approche canadienne, déposé au début de 1987 à un gouvernement conservateur qui lançait le débat sur le rétablissement de la peine de mort, est tombé en porte-à-faux par rapport à l'état des débats sur les politiques pénales

7. Le parti s'est transformé en Parti conservateur. 
au Canada. S'inspirant plutôt d'une perspective rétributiviste, la Commission $^{8}$ était très critique des sanctions "préventives» basées sur l'évaluation des risques que représenterait le contrevenant dans le futur. Elle continuait de mettre de l'avant le principe de modération en favorisant les mesures non carcérales, tout en proposant des lignes directrices en matière de détermination de la peine dans lesquelles les peines maximales seraient beaucoup moins élevées qu'à ce moment-là. Pour le moins qu'on puisse dire, elle aura peu d'impact sur les réformes législatives.

Quelques années plus tard, en août 1988, c'est un comité de la Chambre des communes, composé de députés - le Comité permanent de la Justice et du Solliciteur général (Daubney, 1988) - qui dépose son rapport intitulé Des responsabilités à assumer et qui donne le ton à un certain nombre de réformes pénales. Ayant «noté une baisse de confiance du public envers la justice pénale en général, et en particulier envers la détermination de la peine, le secteur correctionnel et le régime de la mise en liberté sous condition» (p. 9), «le comité estime que la confiance du public envers la justice pénale exige que la protection de la société soit considérée comme l'objet fondamental de chacun de ses éléments. À cet égard, la détermination de la peine ne fait pas exception» (p. 51). Le comité recommande une réforme en profondeur de la mise en liberté sous condition, une augmentation de la période d'inadmissibilité à la libération conditionnelle pour certains délinquants, dont les délinquants sexuels, et plusieurs autres mesures plus sévères pour les délinquants violents, tout en considérant que les solutions de rechange à l'incarcération et les sanctions intermédiaires sont des mesures nécessaires.

En juillet 1990, le gouvernement fédéral présentait une série de documents de consultation, Vers une réforme, qui constituaient la réponse du gouvernement au rapport Daubney et, disait-on, au rapport Archambault. Les propositions visaient, entre autres, à modifier la loi sur la libération conditionnelle de façon, d'une part, que le risque de récidive et la protection de la société soient les principaux critères de décision et, d'autre part, que les délinquants incarcérés pour la première fois dans un pénitencier et qui n'avaient pas commis de crimes violents fassent l'objet d'un examen simplifié avant leur date d'admissibilité à la libération

8. Voir Brodeur (1998). 
conditionnelle, qui survient au tiers de leur peine. Les consultations ont précédé le dépôt en 1992 de la Loi sur le système correctionnel et la mise en liberté sous condition 9 .

Depuis les années 1990 aucun autre rapport important d'orientation des politiques pénales et correctionnelles n'a été produit au palier fédéral ou au Québec. Les politiques et les productions législatives ont plutôt été d'inspiration populiste ${ }^{10}$.

Par ailleurs, c'est au cours des années 1980 que s'est articulé un discours au sujet des victimes d'actes criminels. Tout d'abord, en 1983, le Groupe d'étude fédéral-provincial canadien sur la justice pour les victimes d'actes criminels examine la place de la victime dans le système de justice pénal et fait des recommandations pour améliorer les services et répondre aux besoins des victimes. Puis, en 1987, le Solliciteur général du Canada publie un document de travail, La victime et le système correctionnel, dans lequel il reconnaît qu'on doit répondre aux attentes des victimes dans le domaine correctionnel. Ces rapports illustrent bien une nouvelle préoccupation dans le domaine de la justice pénale et la place de plus en plus grande que prendront les victimes dans le discours pénal ${ }^{11}$.

\section{Les transformations législatives}

\section{Les années 1950 à 1980}

En février 1959, à la suite des recommandations du Comité Fauteux, la Loi relative à la libération conditionnelle de personnes purgeant des sentences d'emprisonnement entre en vigueur. Elle définit le régime de remise en liberté tel qu'on le connaît aujourd'hui et crée la Commission nationale des libérations conditionnelles. Cette loi est modifiée en 1969 et instaure, entre autres, comme suite aux recommandations du Comité Ouimet, la surveillance obligatoire des détenus qui n'ont pas bénéficié de la libération conditionnelle. D'autres modifications au Code criminel, dont l'abolition des châtiments corporels comme peine, viendront aussi concrétiser des propositions de ce comité.

En 1976, le Canada accomplit un geste symbolique ${ }^{12}$ important en abolissant la peine de mort. Malheureusement, pour faire adopter cette

9. L.C. 1992 , ch. 20.

10. Voir Roberts et al. (2003).

11. Voir Gaudreault $(2001$, b).

12. La dernière exécution avait eu lieu en 1962. 
mesure, le gouvernement avait cru bon de faire des compromis importants. La loi subordonnait, en effet, le bénéfice de la libération conditionnelle en cas de condamnation à l'emprisonnement à perpétuité, pour meurtre au premier degré, à l'accomplissement d'au moins vingt-cinq ans de la peine et d'au moins dix ans dans le cas de meurtre au second degré. Cette mesure aura un impact important sur la population carcérale (Landreville, 1995).

On doit aussi mentionner le rapatriement de la Constitution canadienne et l'adoption de la Loi constitutionnelle de $1982^{13}$. Cette loi incorpore la Charte canadienne des droits et libertés dans la Constitution et garantit ainsi à tous les Canadiens certaines libertés et certains droits fondamentaux. La Charte a eu un impact considérable sur le droit criminel canadien.

Enfin, il faut mentionner que vers la fin de cette période, en février 1984, le gouvernement déposa un projet de loi (C-19) modifiant le Code criminel qui venait concrétiser, d'une certaine manière, plusieurs des propositions de la Commission de réforme du droit du Canada. Le projet avait entre autres pour but de «bâtir de nouvelles stratégies de détermination de la peine fondées sur l'utilisation plus fréquente de sanctions non carcérales» (Canada, 1984: 55). En plus de proposer un énoncé de l'objet et des principes de la détermination de la peine, le projet de loi comprenait une gamme plus étendue de solutions de rechange à l'incarcération. On y retrouvait, par exemple, de nouvelles mesures telles le dédommagement, la confiscation et les travaux communautaires, ainsi que des amendements au Code criminel visant à éliminer presque toutes les restrictions à l'imposition de l'amende. Le projet de loi est mort au feuilleton, à la suite du déclenchement des élections. Le Parti libéral perdra le pouvoir au profit des conservateurs, marquant, d'une certaine façon, un point tournant dans l'orientation des politiques pénales canadiennes.

$\mathrm{Au}$ Québec, la période de la «révolution tranquille» en a aussi été une de modernisation de l'administration de la justice pénale, particulièrement à la suite du rapport Prévost (1968). C'est avec la création du ministère de la Justice du Québec, en 1965, que s'amorce la transformation de la justice. Cette année-là, on institue le Service des prisons, suivi en 1967 du Service de probation ${ }^{14}$. La Loi de la probation et des

13. L.R.C. 1985 , app. II, $n^{\circ} 44$, ann. B.

14. Voir Gauthier (1896). 
établissements de détention ${ }^{15}$ vient en 1969 chapeauter l'ensemble des activités dans ce domaine. Par ailleurs, la Loi sur la police de 1968 donne lieu à la création de la Commission de police et de l'Institut de police. Le Parlement québécois innove en adoptant, en 1971, la Loi de l'indemnisation des victimes d'actes criminels ${ }^{16}$ et, en 1972, la Loi de l'aide juridique ${ }^{17}$. En 1975, la création du Comité de protection de la jeunesse inaugure une série de transformations dans le champ des interventions auprès des enfants, dont la Loi sur la protection de la jeunesse ${ }^{18}$ promulguée en 1977 (Trépanier, 1986). Enfin, l'adoption de la Charte des droits et libertés de la personne ${ }^{19}$ constitue une étape importante dans l'histoire de la justice au Québec.

\section{Depuis les années 1980 jusqu'à aujourd'hui}

Cette période est marquée par une activité législative assez intense dans le domaine pénal. Bien que cette production soit souvent en réaction à des événements très médiatisés, à des décisions des tribunaux supérieurs ou à des pressions politiques, qu'elle soit même parfois assez improvisée et manque de cohérence, il est cependant possible d'en dégager des lignes de force.

Tout d'abord, deux pièces majeures sont l'aboutissement de longues discussions: la Loi sur le système correctionnel et la mise en liberté sous condition ${ }^{20}(L S C M L C)$ et la Loi modifiant le Code criminel (détermination de la peine) et d'autres lois en conséquence ${ }^{21}$. La première, comme nous venons de le mentionner, fait suite au rapport Daubney. D'une part, la loi établit que la protection de la société est le critère prépondérant de la remise en liberté sous condition ${ }^{22}$, et permet, par exemple, aux juges de fixer à la moitié de la peine la date d'admissibilité à la libération conditionnelle des criminels violents. D'autre part, elle instaure une procédure d'examen expéditif (PEE), sans audience de libération conditionnelle, pour certains délinquants qui en sont à leur première

\footnotetext{
15. L.Q. 1969, ch. 21.

16. L.R.Q., c.1-6.

17. L.Q. 1972 , ch. 14

18. L.R.Q., c. P-34.1.

19. L.Q. 1975, ch. 6.

20. L.C. 1992 , ch. 20.

21. L.C. 1995 , ch. 22.

22. Voir Robert (2001).
} 
incarcération dans un pénitencier. S'il n'existe aucun motif raisonnable de croire que le délinquant commettra une infraction accompagnée de violence avant la fin de sa peine, la Commission des libérations conditionnelles doit ordonner sa mise en liberté. Enfin, il faut mentionner qu'à l'article quatre elle incorpore dans une loi la reconnaissance des droits des personnes incarcérées: «[L]e délinquant continue à jouir des droits et privilèges reconnus à tous les citoyens, sauf de ceux dont la suppression ou restriction est une conséquence nécessaire de la peine qui lui est infligée.» Cette loi fait aussi, pour la première fois, une place à la victime dans le système correctionnel, précise ses droits et les modalités de sa participation à ce processus ${ }^{23}$.

La loi sur la détermination de la peine est aussi le fruit d'une longue gestation, puisqu'elle est, dans une certaine mesure, une version très édulcorée ${ }^{24}$ du projet de loi C-19 de 1984 à la lumière des débats et des tensions de la décennie qui a suivi. La loi reflète biens ces orientations divergentes. D'une part, elle énonce dans les principes directeurs une présomption en faveur des mesures communautaires ${ }^{25}$. D'autre part, elle déçoit ${ }^{26}$ énormément en ce que, contrairement au projet de loi C-19, elle crée un flou incroyable dans les objectifs du prononcé de la peine en énonçant, sans établir quelque priorité que ce soit, tous les objectifs possibles de la peine et elle est beaucoup moins ferme au sujet du principe de modération. Elle élude presque complètement la question des lignes directrices pour guider le choix des différentes peines, elle maintient que les travaux communautaires sont une condition de la probation plutôt que d'être une peine en soi, elle «durcit» les peines intermédiaires, tout en créant l'emprisonnement avec sursis, peine plus sévère que la probation, sans garantie qu'il sera une réelle solution de rechange à l'incarcération.

$\mathrm{Au}$ moins quatre autres lois visent à raffermir les mesures à l'endroit des délinquants qui ont commis des infractions de violence (en particulier des agressions sexuelles) ou des infractions relatives aux drogues.

23. Voir Gaudreault (2001a).

24. Contrairement à ce que laissent entendre Hatt et al. (1992), cette loi n'est pas qu'une réactualisation du projet $\mathrm{C}-19$.

25. Art. 718.2 «Le tribunal détermine la peine à infliger compte tenu [...] des principes suivants: [...] d) l'obligation, avant d'envisager la privation de liberté, d'examiner la possibilité de sanctions moins contraignantes lorsque les circonstances le justifient.»

26. Des critiques semblables ont déjà été formulées par plusieurs, dont Madame Hélène Dumont et Monsieur Jean-Paul Brodeur, lors du colloque de l'Institut canadien d'administration de la justice pénale, tenu à Montréal en avril 1997. 
En 1986, le projet de loi C-67 autorise la Commission des libérations conditionnelles à maintenir en incarcération un délinquant jusqu'à l'expiration de sa peine s'il y a «des motifs raisonnables de croire que le délinquant commettra vraisemblablement, avant l'expiration de sa peine, une infraction causant la mort ou un dommage grave». Auparavant, les détenus qui n'avaient pas obtenu une libération conditionnelle étaient libérés sous surveillance aux deux tiers de leur peine.

En 1996, l'adoption de la Loi réglementant certaines drogues et autres substances $^{27}$ s'écarte, comme l'a souligné Brodeur (1999), de certains principes des politiques pénales canadiennes. En particulier, on y énonce une liste de circonstances aggravantes dans la commission de certaines infractions concernant les drogues que le tribunal doit prendre en considération lors de la détermination de la peine. La loi stipule de plus que:

10 (3) Le tribunal qui décide de n'imposer aucune peine d'emprisonnement à la personne visée au paragraphe (1), bien qu'il soit convaincu de l'existence d'une ou de plusieurs circonstances aggravantes mentionnées à ce paragraphe, est tenu de motiver sa décision.

Même si le Code criminel prévoyait déjà que le juge devait motiver sa décision lors du prononcé de la peine ${ }^{28}$, ce type de présomption d'incarcération est tout à fait nouveau dans le droit canadien.

La même année, à la suite de fortes pressions de l'opposition, le Parlement a adopté le projet de loi C-45 qui modifiait l'article $745 \mathrm{du}$ Code criminel concernant la révision judiciaire de l'inadmissibilité à la libération conditionnelle des personnes condamnées pour meurtre. En 1976, lors de l'abolition de la peine de mort, la peine pour meurtre au premier degré est devenue l'emprisonnement à perpétuité et le bénéfice de la libération conditionnelle était subordonné, dans ce cas, à l'accomplissement d'au moins vingt-cinq ans de la peine. Néanmoins, le condamné qui avait purgé au moins quinze ans de sa peine pouvait demander une révision judiciaire du délai préalable à sa libération conditionnelle. Le projet de loi C-45 nie aux personnes déclarées coupables de plus d'un meurtre le droit d'invoquer l'article 745, crée un mécanisme d'examen préalable des demandes et exige l'unanimité du jury (au lieu de l'assentiment d'au moins les deux tiers des membres) 
pour réduire le délai d'inadmissibilité. Cette loi restreint donc considérablement la portée de la révision judiciaire.

Le projet de loi C-55, entré en vigueur en $1997^{29}$, vise lui aussi les délinquants dits dangereux. Entre autres, d'une part, il renforce de plusieurs façons les dispositions de la partie XxIv du Code criminel qui prévoit une peine d'emprisonnement pour une période indéterminée pour les délinquants déclarés délinquants dangereux. D’autre part, il crée une nouvelle catégorie de délinquants «représentant un risque élevé de récidive», les «délinquants à contrôler», soit certains délinquants sexuels qui seront soumis à une ordonnance de surveillance pour une période maximale de dix ans, après avoir purgé la peine imposée pour l'infraction pour laquelle ils ont été déclarés coupables.

Deux autres lois se sont fait remarquer parce qu'elles imposent des peines minimales très élevées à l'encontre de l'avis de plusieurs commissions, dont la Commission Archambault, qui recommandait plutôt l'abolition des peines minimales, et contrairement à la situation légale canadienne où ces peines sont exceptionnelles. Tout d'abord, le projet de loi C-68 concernant les armes à feu ${ }^{30}$, qui prévoit une peine minimale de quatre ans pour une dizaine d'infractions sérieuses au Code criminel, lorsqu'elles ont été commises avec une arme à feu ${ }^{31}$. Cette peine minimale est très importante si l'on considère qu'avant cette modification, l'homicide involontaire, qui était dans la pratique l'infraction la plus punie après le meurtre, entraînait une peine médiane de quatre ${ }^{32}$ ou cinq ${ }^{33}$ ans. Le projet de loi C-27 de $1997^{34}$ crée, quant à lui, une peine minimale de cinq ans pour ceux qui vivent des produits de la prostitution d'une personne âgée de moins de dix-huit ans.

En 1997, le Parlement a aussi voté une loi «antigangs » ${ }^{35}$ qui édicte que la participation aux activités d'un gang par la perpétration d'actes criminels au profit du gang ou par le fait de les favoriser constitue une infraction. Il prévoit aussi que, indépendamment du fait qu'un meurtre ait été commis avec préméditation, le meurtre commis à l'aide d'explosifs est un meurtre au premier degré s'il a été commis en relation avec

29. L.C. 1997 , ch. 17.

30. L.C. 1995 , ch. 39.

31. Pour une critique de ces amendements, voir Dumont (1997).

32. Turner (1993), tableau 8.

33. Archambault (1987), p. 575.

34. L.C. 1997, ch. 16.

35. L.C. 1997, ch. 23. 
un gang. Un contrevenant déclaré coupable d'une infraction ayant été commise en relation avec un gang serait passible d'une peine plus élevée ou d'une période plus longue avant d'être admissible à la libération conditionnelle.

Plusieurs autres projets de loi portant sur des infractions spécifiques ont été adoptés pendant cette période. Selon une analyse réalisée par Brodeur (1999), la tendance générale qui s'en dégage est un accroissement de la punition, soit par l'augmentation des peines maximales prévues au Code criminel, soit par la création de nouvelles infractions, l'augmentation des peines étant particulièrement évidente concernant les infractions à caractère sexuel.

La question du crime organisé et celle du terrorisme ont donné lieu à l'adoption de nouvelles lois, Loi sur le crime organisé36 et Loi sur le recyclage des produits de la criminalité et le financement des activités terroristes ${ }^{37}$ «[...] dont l'adoption faisait l'objet de beaucoup de controverses» (Boisvert et Dumont, 2004: 205). Selon ces mêmes auteures:

Ces lois créent de nouvelles infractions à très large portée. Celles-ci se construisent sur les notions de complot et de participation criminelle, de même que sur certaines infractions préliminaires comme la tentative. Par conséquent, elles s'approchent dangereusement de la notion «criminalité par association». [...] Il faut déplorer ce recours au flou et au vague dans la rédaction des crimes et le danger que cela recèle de possibles accrocs au principe de légalité en droit pénal canadien. (p. 206)

Dans ce même article, les deux professeures de droit font aussi part de leur inquiétude «devant l'augmentation spectaculaire des pouvoirs de police consentis sous prétexte d'assurer la sécurité de la population» (p. 206).

Pour satisfaire aux pressions d'une certaine opinion publique venant de l'Ouest, le gouvernement a adopté en 2002 la Loi concernant le système de justice pénale pour les adolescents ${ }^{38}$, entrée en vigueur en 2003. Cette loi qui met l'accent sur la protection de la société, favorise, entre autres, l'imposition de peines normalement applicables aux adultes à des adolescents. C'est une autre mesure législative visant à augmenter la sévérité des lois.

Le nouveau gouvernement conservateur de M. Harper a en 2006 accentué cette tendance en faisant preuve d'une fureur répressive peu

36. L.C. 2001, c. 32.

37. L.C. 2000 , c. 17.

38. L.C. 2002 , c. 1. 
commune. Il a déposé au moins onze projets de loi modifiant le Code criminel visant à créer de nouvelles infractions, à augmenter les peines minimales ou maximales, à donner de nouveaux pouvoirs aux agents de la paix et à restreindre le pouvoir discrétionnaire du poursuivant ou du tribunal.

On ne peut passer sous silence son intention de créer au Canada une forme de disposition que l'on nomme «three strikes» aux États-Unis. Cette disposition permettrait de déclarer délinquants dangereux, et de leur imposer une peine d'emprisonnement pour une période indéterminée, certaines personnes reconnues coupables d'une troisième infraction leur ayant valu des peines d'emprisonnement de deux ans et plus. Le même projet de loi C-27 vise aussi à modifier l'article 810 du Code criminel pour permettre que la durée d'un engagement de ne pas troubler l'ordre public (imposé à une personne qui n'a pas été reconnue coupable) soit de deux ans (au lieu d'un an) et que cet engagement puisse être assorti de l'obligation de porter un dispositif de surveillance à distance (surveillance électronique). Le projet de loi C-35, quant à lui, prévoit que le prévenu inculpé de certaines infractions graves où une arme à feu a été utilisée devra prouver que sa détention avant le procès n'est pas justifiée. Ce renversement du fardeau de la preuve illustre le peu de cas qu'on fait de la présomption d'innocence et l'escalade des mesures répressives en droit pénal.

Ces projets de loi démontrent bien l'orientation populiste des politiques pénales des dernières décennies qui s'est accentuée avec l'élection d'un gouvernement conservateur. Ces politiques et les lois qui en découlent ne reposent plus sur des analyses objectives de la réalité, font fi des résultats de recherche au sujet de l'efficacité et des effets discriminatoires de ces mesures, ainsi que des principes de justice et de modération en droit pénal. Elles privilégient surtout le recours à l'emprisonnement pour répondre aux pressions d'une certaine opinion publique et pour augmenter le capital politique du parti au pouvoir.

\section{Les transformations des pratiques pénales}

Les infractions rapportées à la police et les affaires traitées par les tribunaux

Un des faits les plus marquants du dernier demi-siècle à propos de la criminalité et de la répression pénale est l'augmentation considérable 
des infractions enregistrées par la police. L'augmentation de ces infractions est générale dans les pays occidentaux. En Grande-Bretagne, par exemple, Garland (2001: 90) rapporte qu'elles sont passées de 1 pour 100 habitants, à 10 pour 100 habitants de 1950 à 1994 . Aux ÉtatsUnis, cette augmentation est aussi très rapide de 1960 à 1980, puis les taux se stabilisent dans les années 1980, pour diminuer à partir du début des années 1990.

Au Canada, la courbe de l'évolution des infractions enregistrées par la police est relativement semblable, mais à une autre échelle, à celle des États-Unis. De 1962 à 1991, il y a une forte augmentation de toutes les infractions au Code criminel. Les taux par 100000 habitants se multiplient par 3,7 suivis d'une diminution sensible de $25 \%$ de 1992 à 2005 , de sorte qu'en 2005, il y avait un taux d'infractions enregistrées 2,8 fois plus élevé qu'en 1962.

La fluctuation n'a pas été la même pour les infractions de violence que pour celles contre les biens. Le taux des infractions de violence enregistrées par la police s'est multiplié par 4,9 de 1962 à 1992, pour diminuer de $13 \%$ jusqu'en 2005. Dans le cas des infractions contre les biens, l'augmentation a été moins spectaculaire; elles se sont multipliées par 3,3 et ont connu une forte diminution de 39\% depuis 1991 (Gannon, 2005). Cette diminution remarquable, semblable à celle des États-Unis (Ouimet, 2002; 2004), s'est produite dans plusieurs catégories d'infractions. De 1991 à 1999, il y a eu une diminution de $43 \%$ des homicides, de $31 \%$ des agressions sexuelles, de $23 \%$ des voies de fait et des vols qualifiés et de $35 \%$ des cambriolages (Ouimet, 2002: 41).

Au Canada, on constate aussi un très faible taux d'élucidation des infractions contre les biens, mais surtout une nette diminution de ce taux depuis le début des années 1960. Le taux d'élucidation ${ }^{39}$ de toutes les infractions contre les biens est passé de $22 \%$, en 1962, à $14 \%$, en 2003 ; celui des cambriolages, de 20 à $10 \%$, et celui des vols de véhicules, de 23 à $8 \%$. Par contre, le taux d'élucidation des infractions de violence est demeuré stable, passant de 46 à $49 \%$.

Les affaires traitées par les tribunaux semblent aussi accorder de moins en moins de place aux prédations en faveur des infractions de violence. Si les infractions contre les biens représentaient $40 \%$ des condamnations en 1962 , elles ne comptent plus que pour $30 \%$ des condamnations en

39. «Solution par mise en accusation». 
1999-2000. Par contre, les condamnations pour des infractions contre les personnes sont passées de 11 à $20 \%$.

\section{L'incarcération}

Cette attention portée aux infractions de violence au détriment des prédations se constate aussi sur le plan des peines. Les personnes condamnées à des peines de deux ans et plus pour des infractions de violence constituent une proportion de plus en plus grande des personnes admises dans les pénitenciers canadiens, alors qu'il n'y en a presque plus qui sont admises pour des prédations (tableau 1).

L'augmentation la plus spectaculaire dans le pourcentage des admissions, selon les infractions, se produit pour les voies de fait, qui passent de $1,4 \%$ des admissions en 1964-1965, à 12,6\% des admissions en 2003-2004. On constate aussi une augmentation dans le pourcentage des homicides, qui a plus que doublé (2,5 à 5,7\%), et des agressions sexuelles ${ }^{40}$, qui se sont presque multipliées par deux (7,2 à 10,8\%). Par contre, les introductions par effraction sont passées de 30,6\% des admissions à $14 \%$, et les vols, fraudes et possession de biens volés ont diminué de 25,6 à 7,9\%. Somme toute, si les personnes condamnées à des peines de deux ans et plus pour des infractions contre les biens représentaient $57 \%$ des admissions dans les pénitenciers en 1964-1965, elles ne forment plus que $22 \%$ des admissions en 2003-2004. C'est une diminution très remarquable.

TAB LEA U 1

Admissions dans les pénitenciers canadiens selon le type d'infraction 1964-1965; 2003-2004

\begin{tabular}{|l|c|c|c|c|}
\hline & \multicolumn{2}{|c|}{$\mathbf{1 9 6 4 - 1 9 6 5}$} & \multicolumn{2}{c|}{$\mathbf{2 0 0 3 - 2 0 0 4}$} \\
\hline Infractions & $\mathbf{N}$ & $\mathbf{\%}$ & $\mathbf{N}$ & $\%$ \\
\hline Homicides & 93 & 2,5 & 243 & 5,7 \\
\hline Vols qualifiés & 601 & 16,2 & 813 & 19,2 \\
\hline Agressions sexuelles & 265 & 7,2 & 456 & 10,8 \\
\hline Voies de fait & 53 & 1,4 & 532 & 12,6 \\
\hline Introductions par effraction & 1129 & 30,6 & 591 & 14 \\
\hline Vols, fraudes, possession de biens volés & 945 & 25,6 & 333 & 7,9 \\
\hline Infractions relatives aux drogues & 74 & 2 & 584 & 13,8 \\
\hline TOTAL & 3693 & 100 & 4226 & \\
\hline
\end{tabular}

Sources: Statistique Canada - Catalogue 85-207 (1968-1969); catalogue 85-211 (2003-2004).

40. Il faut noter que les catégories de ces infractions se sont considérablement modifiées pendant cette période. 
La durée des peines d'incarcération de plus de deux ans a tendance à diminuer au moins depuis 1985 (tableau 2). Le pourcentage des personnes admises pour des peines de moins de trois ans est passé de 40\% en 1985-1986 à 55\% en 2003-2004. Celui des personnes admises pour des peines de trois à quatre ans est passé de $26 \%$ en 1985-1986 à 20\% en 2003-2004. Toutes les catégories de peine de quatre ans et plus, sauf celles de dix ans et plus, ont diminué durant cette période. Cela tend à démontrer que la loi de $1995^{41}$ qui prévoyait une peine minimale consécutive de quatre ans pour certaines infractions commises au moyen d'une arme à feu a eu peu d'impact.

TABLEA U 2

\section{Admissions dans les pénitenciers canadiens par peine globale} de 1985-1986 à 2003-2004

\begin{tabular}{|c|c|c|c|c|c|c|c|c|c|c|c|c|c|c|c|}
\hline \multirow[t]{2}{*}{ Année } & \multicolumn{2}{|c|}{$<3$ ans } & \multicolumn{2}{|c|}{$3<4$ ans } & \multicolumn{2}{|c|}{$4<5$ ans } & \multicolumn{2}{|c|}{$5<6$ ans } & \multicolumn{2}{|c|}{$6<7$ ans } & \multicolumn{2}{|c|}{$7<10$ ans } & \multicolumn{2}{|c|}{10 et +} & \multirow[t]{2}{*}{ Total } \\
\hline & $\mathrm{N}$ & $\%$ & $\mathrm{~N}$ & $\%$ & $\mathrm{~N}$ & $\%$ & $\mathrm{~N}$ & $\%$ & $\mathrm{~N}$ & $\%$ & $\mathrm{~N}$ & $\%$ & $\mathrm{~N}$ & $\%$ & \\
\hline $1985-86$ & 1462 & 40,1 & 938 & 25,7 & 449 & 12,3 & 285 & 7,8 & 132 & 3,6 & 242 & 6,6 & 136 & 3,7 & 3644 \\
\hline $1986-87$ & 1477 & 40,2 & 921 & 25,1 & 487 & 13,3 & 260 & 7,1 & 129 & 3,5 & 243 & 6,6 & 155 & 4,2 & 3672 \\
\hline $1987-88$ & 1458 & 40,2 & 898 & 24,8 & 460 & 12,7 & 281 & 7,7 & 147 & 4,1 & 220 & 6,1 & 162 & 4,5 & 3626 \\
\hline $1988-89$ & 1524 & 41,0 & 933 & 25,1 & 438 & 11,8 & 276 & 7,4 & 145 & 3,9 & 242 & 6,5 & 157 & 4,2 & 3715 \\
\hline $1989-90$ & 1644 & 41,1 & 1040 & 26,0 & 506 & 12,6 & 282 & 7,0 & 173 & 4,3 & 223 & 5,6 & 135 & 3,4 & 4004 \\
\hline $1990-91$ & 1588 & 39,7 & 1018 & 25,4 & 540 & 13,5 & 317 & 7,9 & 166 & 4,1 & 226 & 5,6 & 150 & 3,7 & 4005 \\
\hline 1991-92 & 1928 & 42,0 & 1191 & 25,9 & 552 & 12,0 & 337 & 7,3 & 171 & 3,7 & 291 & 6,3 & 148 & 3,2 & 4590 \\
\hline $1992-93$ & 2050 & 42,1 & 1190 & 24,4 & 640 & 13,1 & 340 & 7,0 & 188 & 3,9 & 295 & 6,1 & 166 & 3,4 & 4869 \\
\hline $1993-94$ & 2037 & 41,2 & 1281 & 25,9 & 603 & 12,2 & 363 & 7,3 & 214 & 4,3 & 284 & 5,7 & 166 & 3,4 & 4948 \\
\hline 1994-95 & 2041 & 42,8 & 1165 & 24,4 & 558 & 11,7 & 327 & 6,9 & 199 & 4,2 & 295 & 6,2 & 182 & 3,8 & 4767 \\
\hline $1995-96$ & 1783 & 40,5 & 1008 & 22,9 & 541 & 12,3 & 321 & 7,3 & 163 & 3,7 & 247 & 5,6 & 343 & 7,8 & 4402 \\
\hline $1996-97$ & 1823 & 39,9 & 1019 & 22,3 & 617 & 13,5 & 334 & 7,3 & 183 & 4,0 & 270 & 5,9 & 352 & 7,7 & 4569 \\
\hline $1997-98$ & 1840 & 41,7 & 927 & 21,0 & 587 & 13,3 & 265 & 6,0 & 176 & 4,0 & 269 & 6,1 & 322 & 7,3 & 4412 \\
\hline 1998-99 & 1877 & 40,7 & 992 & 21,5 & 632 & 13,7 & 364 & 7,9 & 217 & 4,7 & 254 & 5,5 & 277 & 6,0 & 4612 \\
\hline 1999-00 & 1861 & 43,0 & 965 & 22,3 & 524 & 12,1 & 299 & 6,9 & 156 & 3,6 & 234 & 5,4 & 281 & 6,5 & 4329 \\
\hline $2000-01$ & 1995 & 46,7 & 914 & 21,4 & 530 & 12,4 & 273 & 6,4 & 141 & 3,3 & 167 & 3,9 & 256 & 6,0 & 4272 \\
\hline 2001-02 & 1861 & 45,2 & 935 & 22,7 & 465 & 11,3 & 268 & 6,5 & 161 & 3,9 & 189 & 4,6 & 239 & 5,8 & 4118 \\
\hline $2002-03$ & 2183 & 51,0 & 908 & 21,2 & 381 & 8,9 & 253 & 5,9 & 146 & 3,4 & 154 & 3,6 & 257 & 6,0 & 4281 \\
\hline 2003-04 & 2324 & 55,0 & 845 & 20,0 & 380 & 9,0 & 203 & 4,8 & 118 & 2,8 & 139 & 3,3 & 216 & 5,1 & 4226 \\
\hline
\end{tabular}

Sources: 1985 à 1995: Boe, R., Motiuk, L., et Muirhead, M. (1998). Nouvelles tendances et caractéristiques relatives à la population carcérale au Canada: 1983-1984 à 1996-1997. Direction générale de la recherche, Service correctionnel du Canada. http://www.csc-scc.gc.ca. 1995 à 1998: Services correctionnels pour adultes au Canada, 1997-1998, tableau 39, Statistique Canada n 85-811. 1998 à 2001: Services correctionnels pour adultes au Canada, 2000-2001, tableau 29, Statistique Canada n 85-211. 2001 à 2004: Services correctionnels pour adultes au Canada, 2003-2004, tableau 29, Statistique Canada, $n^{\circ}$ 85-211. 
Soulignons aussi que cette population des pénitenciers vieillit considérablement. Au Québec, par exemple, si les moins de 25 ans représentaient 58\% des admissions dans les pénitenciers en 1975, en 1999 ils ne représentaient plus que $18 \%$ des admissions. Par contre, les personnes âgées de 35 ans et plus, qui représentaient $13 \%$ des admissions en 1975, en représentaient 47\% en 1999 (Landreville, 2001). Cette transformation radicale s'explique en partie par les changements démographiques, mais aussi par une transformation de la réaction pénale: on inflige de moins en moins de peines de plus de deux ans aux jeunes adultes qui en sont à leur première condamnation et il y a des peines de plus en plus sévères pour les infracteurs sexuels, particulièrement ceux qui sont condamnés pour la première fois après l'âge de 35 ans. Il y a aussi une augmentation de condamnations à des peines supérieures à deux ans pour les délinquants sur le tard (âgés de 35 ans et plus) condamnés au pénitencier pour une première infraction relative au trafic de stupéfiants.

Mais on doit cependant constater que, malgré les discours justificateurs sur le changement, sur la réhabilitation des personnes incarcérées ou sur la neutralisation des «dangereux», la prison continue de jouer le rôle des hôpitaux généraux et des dépôts de mendicité dans la gestion quotidienne des pauvres, des vagabonds, des prostituées, des groupes marginalisés et de certains malades mentaux.

Au Canada comme ailleurs, la prison s'adresse surtout à ces groupes ou fractions de classes les plus défavorisés. Plus de 200000 personnes sont incarcérées chaque année et environ la moitié d'entre elles le sont pour une période de moins d'un mois. Parmi les condamnés admis pour des peines de moins de deux ans, de 25 à $45 \%$, selon les provinces, le sont pour défaut de payer des amendes, de 10 à $33 \%$ pour avoir conduit lorsque leurs capacités étaient affaiblies, et seulement de 8 à $25 \%$ pour des infractions de violence. De plus, dans certaines provinces - les provinces centrales -, de 33 à $80 \%$ des personnes admises dans les établissements provinciaux sont des autochtones. Ce groupe, qui représente environ $3 \%$ de la population canadienne, constitue $« 12 \%$ des délinquants sous responsabilité fédérale et $15 \%$ de la population carcérale (des pénitenciers), au 31 mars 1997» (Canada, 1998: xxix) ${ }^{42}$. En 2003-2004, les autochtones représentaient 54\% des personnes

42. Voir aussi Roberts et Doob (1997). 
admises dans les pénitenciers du Manitoba et $63 \%$ de celles admises en Saskatchewan ${ }^{43}$.

Au Canada, les taux de détention des adultes par 100000 habitants sont demeurés relativement stables durant le dernier demi-siècle. Ils ont varié entre 90 et 100 de 1950 à 1965 pour redescendre sous 85 au début des années 1970, et remonter graduellement à 115 en 1996, et redescendre de nouveau à 100 actuellement. Cette stabilité est particulièrement remarquable comparativement à l'augmentation qui s'est produite chez la plupart des pays occidentaux et particulièrement chez nos voisins du sud.

En Europe, les Pays-Bas, qui était un pays modèle, a vu son taux de détention multiplié par 4,4 depuis 1983, passant de 28 à plus de 123 en 2004. En Angleterre et au Pays de Galles, le taux est passé de 87 en 1983 à 140; au Portugal, il a fait un bond de 59 à 137; tandis qu'en France, il passait de 70 à 96 durant la même période. La majorité des autres pays du Conseil de l'Europe ont connu une augmentation de l'ordre de 15 à $25 \%$ durant la dernière décennie et quelques-uns, dont la Finlande et l'Allemagne, ont vu leur taux légèrement augmenter après une diminution très prononcée. Le «tout-carcéral» s'est surtout réalisé aux États-Unis, où les taux de détention ont connu une progression fulgurante depuis 1970. Ils sont passés de 175 en 1970 à 212 en 1980 , 313 en 1985, 645 en 1997, et à plus de 750 au 30 juin $2006^{44}$.

En ce qui concerne la remise en liberté des personnes incarcérées, il y a une diminution marquée des remises en liberté sous une forme ou une autre de libération conditionnelle et conséquemment une augmentation des personnes libérées en libération d'office aux deux tiers de leur sentence ou à la fin de leur sentence depuis les dix dernières années. Au Canada, le pourcentage des détenus fédéraux libérés en libération d'office est passé de $41 \%$ en 1993-1994 à $48 \%$ en 1994-1995 pour atteindre $65 \%$ en 2003-2004 ${ }^{45}$. Au Québec, le pourcentage des détenus des établissements de détention provinciaux libérés en libération conditionnelle, par rapport aux personnes admissibles à une telle libération, a chuté de $72 \%$ en $1993-1994$ à $36 \%$ en $2004-2005^{46}$. Puisque les remi-

43. Services correctionnels pour adultes au Canada, 2003-2004, catalogue $\mathrm{n}^{\circ} 85-211$. Ottawa: Statistique Canada.

44. Sabol, Minton et Harrison (2007).

45. Services correctionnels pour adultes au Canada, 2003-2004, catalogue $\mathrm{n}^{\circ} 85-211$. Ottawa: Statistique Canada.

46. Landreville et Charest (2004), p. 39. 
ses en liberté sous condition sont probablement le plus important facteur qui détermine la durée des séjours en détention et par conséquent le stock carcéral (le nombre de personnes incarcérées à un moment donné), il va de soi que le taux de détention aurait diminué au Canada, n'eût été cette chute très importante des remises en liberté sous condition.

\section{Les grandes tendances et les paradoxes de la répression pénale}

\section{Sur le plan des discours de politique pénale}

- Si, des années 1960 à 1985 environ, les commissions et comités d'experts ainsi que les élites politiques mettaient l'accent sur les principes de justice, d'équité et de modération en droit pénal, si l'emprisonnement était vu comme une mesure de dernier ressort, si on favorisait la réhabilitation et l'on préconisait les solutions de rechange à l'incarcération, depuis les vingt dernières années la protection de la société devient le nouveau leitmotiv. Il s'agit surtout maintenant de répondre aux préoccupations des citoyens, de faire la lutte à la criminalité, de neutraliser les délinquants dangereux, particulièrement les «prédateurs sexuels», et, d'une façon démagogique, de prétendre répondre aux préoccupations des victimes en augmentant la sévérité des peines.

\section{Sur le plan législatif}

- Durant les années 1960 et 1970 , on a aboli les châtiments corporels et la peine de mort, on a créé les libérations conditionnelles, et, au Québec, le service de probation et l'aide juridique, tout en adoptant une loi sur l'indemnisation des victimes d'actes criminels.

- Mais en général, surtout depuis les deux dernières décennies, les lois sont devenues de plus en plus sévères.

- On a multiplié les peines minimales ${ }^{47}$ et aussi augmenté les peines maximales.

- On a connu un regain d'intérêt pour les délinquants sexuels. On a multiplié les infractions et augmenté les peines pour les agresseurs sexuels, les pédophiles, la pornographie (infantile), le tourisme sexuel.

- Plus récemment, on a particulièrement ciblé le crime organisé (les gangs criminels) et le terrorisme au moyen d'un train de nouvelles mesures et de pouvoirs plus étendus accordés à la police, qui ont suscité de profondes inquiétudes.

47. Voir Crutcher (2001). 
- D’une façon générale, on a vu se développer une tendance duale ${ }^{48}$ dans les législations. D’une part, en effet, il y a une augmentation de la sévérité des mesures pénales pour ceux qu'on qualifie de délinquants violents, récidivistes, «dangereux», les membres des organisations criminelles, alors que sont apparues des mesures moins draconiennes, ordinairement des solutions de rechange à l'incarcération, pour les délinquants primaires et ceux qui ont commis des infractions contre les biens.

\section{Sur le plan des pratiques}

- Depuis un demi-siècle, il y a eu tout d'abord une augmentation considérable des infractions enregistrées par la police. L'augmentation a été plus marquée pour les infractions de violence. Il y a eu cependant une forte diminution des infractions enregistrées par la police depuis le début des années 1990, particulièrement en ce qui concerne les infractions contre les biens.

- Le taux d'élucidation des prédations est faible et il a chuté considérablement depuis les années 1960.

- Les affaires traitées par les tribunaux semblent aussi accorder de moins en moins de place aux prédations en faveur des infractions de violence.

- Les taux de détention ont augmenté de façon vertigineuse aux États-Unis et d'une façon significative dans la plupart des pays européens, alors qu'ils sont demeurés relativement stables au Canada, quelle que soit l'évolution des infractions enregistrées par la police et le ton répressif des discours et des législations des vingt dernières années.

- Au Canada, on constate aussi une pratique duale dans l'imposition des peines d'emprisonnement. Les peines de plus de deux ans visent maintenant surtout des infractions de violence ou celles relatives aux stupéfiants, alors que les prédations qui comptaient pour plus de $57 \%$ des admissions il y a quarante ans ne représentent plus que $22 \%$ des admissions dans les pénitenciers. Ce constat va dans le sens des recommandations des années 1960-1970 de n'utiliser l'emprisonnement que pour des infractions graves contre la personne.

- La durée des peines d'incarcération de plus de deux ans a tendance à diminuer au moins depuis 1985. Cela tend à démontrer que les discours et les lois augmentant les peines maximales ou instituant des peines minimales, telle la loi de $1995^{49}$ qui prévoyait une peine minimale consécutive de quatre ans pour certaines infractions commises au moyen d'une arme à feu, ont eu peu d'impact.

- Il y a de moins en moins de jeunes de moins de 25 ans admis dans les pénitenciers. La réaction pénale s'est transformée: on utilise de moins en

48. «Bifurcation», selon l'expression de Bottoms (1977).

49. L.C. 1995 , c. 39. 
moins de peines de plus de deux ans pour les jeunes adultes qui en sont à leur première condamnation. Les solutions de rechange à l'incarcération sont probablement aussi de plus en plus utilisées envers les jeunes adultes.

- Enfin, quels que soient les discours de politique pénale et même les modifications législatives, il ne faut pas perdre de vue les pratiques quotidiennes d'enfermement et se rappeler, à la suite de Faugeron et Le Boulaire (1992), que la prison recouvre «[...] une fonction pratique de sûreté caractérisée par sa fréquence, par la rapidité de l'intervention, une mise à l'écart temporaire, des procédures presque automatiques et des justifications essentiellement liées à l'ordre public; le dispositif gère des populations flottantes et peu qualifiées [...].»(p. 7 $)^{50}$

Nous pouvons être d'accord, dans une certaine mesure, avec O'Malley (1999) pour constater que les pratiques de la punition au Canada ont été "volatiles et contradictoires» du moins en ce que, comme nous l'avons démontré, les pratiques effectives sont, particulièrement durant les dernières années, assez différentes des discours politiques et des lois qui ont été adoptées. Cependant, nous croyons que tant les discours politiques que les législations pénales ont pris un «tournant punitif», contrairement à ce qu'il laisse entendre (Meyer et O'Malley, 2005).

\section{Hypothèses pour comprendre ces transformations}

Durant les cinquante dernières années, il y a eu, sans contredit, de profonds changements dans le champ du «contrôle de la criminalité». Cette reconfiguration est le résultat de décisions politiques et administratives qui s'inscrivent dans une transformation des rapports sociaux et, selon Garland (2001), de nouvelles sensibilités culturelles et de perceptions sociales du crime et de son châtiment.

Ces changements dans les discours, les politiques, les législations et les pratiques à propos de la criminalité, de son contrôle et d'une façon plus spécifique de la répression pénale s'inscrivent dans des transformations politiques plus générales et plus profondes au sujet du rôle de l'État, des relations entre les groupes sociaux, de la répartition des ressources. Même si elles participent à ces transformations des visions du monde et des pratiques sociopolitiques, les politiques pénales sont en grande partie influencées par les changements politiques, sociaux et économiques des dernières décennies. 
La culture politique qui mettait l'accent sur la solidarité, la justice sociale, les responsabilités collectives et la régulation de l'économie a de plus en plus fait place à une culture d'exclusion, de responsabilité individuelle et de primauté des lois du marché. Alors qu'on valorisait les politiques sociales pour aider les plus démunis et améliorer le bienêtre de tous et des politiques économiques de répartition des richesses, on préconise de plus en plus un désengagement de l'État dans les domaines social et économique mais une intensification du contrôle des populations pauvres et marginales. Dans plusieurs pays, les politiques conservatrices des années 1980, illustrées par Reagan et Thatcher, ont mené la charge contre les politiques sociales, contre le rôle régulateur de l'État et, dans une certaine mesure, contre la culture dite permissive des années 1960. Les pauvres et les marginaux sont de moins en moins l'objet de la solidarité sociale, mais sont considérés comme des populations «responsables de leurs malheurs » qu' il faut surveiller et contrôler. Même si cette nouvelle culture politique s'est aussi manifestée avec moins d'ampleur au Canada, elle n'en est pas moins présente.

Mais ces changements structuraux, politiques et culturels, ainsi que les nouveaux discours et les nouvelles politiques pénales ont eu des effets différentiels sur les pratiques pénales selon les pays. Si, pour ne citer que l'exemple de nos voisins du sud, celui qui nous intéresse le plus, les États-Unis ont connu des pratiques sentencielles de plus en plus répressives et une augmentation vertigineuse de leur population carcérale - les taux de détention se sont multipliés par quatre depuis 35 ans et sont sept fois plus élevés qu'au Canada - le Canada a eu un taux de détention relativement stable durant cette période.

Ces transformations différentielles des pratiques doivent probablement être expliquées par des facteurs historiques, des structures et des cultures politiques, des systèmes et des traditions juridiques propres à chaque pays. Aux États-Unis, l'histoire esclavagiste et de ségrégation, et la persistance de la perception dévalorisée de l'«autre» afro-américain, peut probablement expliquer en partie le fait qu'on puisse tolérer des taux de punitivité, de contrôle et d'enfermement qui seraient considérés scandaleux presque partout ailleurs ${ }^{51}$. La tradition politique "populiste», selon laquelle les représentants élus (politiciens et même juges, procureurs, shérifs) doivent répondre aux volontés de leurs mandants,

51. Voir Tonry (2005) et Wacquant (2005) à ce sujet. 
n'est pas sans lien avec la rapidité et l'intensité des réponses répressives du dernier quart de siècle.

$\mathrm{Au}$ Canada par contre, nous partageons une culture politique plus «élitiste», où les représentants politiques ne se perçoivent ${ }^{52}$ pas comme de simples exécutants des volontés des électeurs mais considèrent, dans une certaine mesure, qu'ils ont le devoir moral et politique de proposer et de mettre en place des politiques pour le «bien commun». Cette culture pourrait expliquer, en partie, une certaine modération dans les pratiques pénales. De plus, une tradition et une culture d'indépendance de la magistrature, ainsi que l'inclusion d'une charte des droits dans la Constitution assurent au pouvoir judiciaire un rôle de contrepoids non négligeable qui peut freiner un emballement pénal intempestif. Enfin, le fait que dans notre régime constitutionnel le droit criminel est de compétence exclusive du gouvernement central, alors que l'administration de la justice et l'exécution des peines dépendent surtout des provinces, favorise des pratiques pénales variables d'une province à l'autre, complique la coordination des politiques et des pratiques, et rend tout changement rapide ou profond presque impossible.

On a constaté un écart important entre le discours et les lois répressives des dernières décennies et l'application de la loi. Cet écart peut être expliqué, du moins en partie, par la résistance institutionnelle. Si, comme l'ont démontré Artières et Lascoumes (2004) à propos des politiques pénitentiaires, «les institutions ont une forte capacité à résister à des transformations majeures y compris des ruptures politiques» (Lascoumes, 2006: 416), on ne sera pas surpris que de nouvelles orientations punitives, qui heurtent certains principes bien ancrés, l'idéologie de nombreux acteurs des tribunaux et des agences correctionnelles ainsi que des façons de faire, n'aient pas les effets proclamés, du moins à court et à moyen terme. La création de nouvelles infractions, l'augmentation de peines maximales et même l'introduction de peines minimales peuvent rencontrer une certaine résistance de la part de plusieurs acteurs judiciaires qui y voient une entorse à certains principes de droit, une certaine incompatibilité par rapport à la Charte des droits, un empiètement sur leur pouvoir discrétionnaire ou encore une entrave à leur façon de faire quotidienne (la négociation de plaidoyer, par exemple). Par ailleurs, comme le soutient O'Malley (2006), «[au Canada] le

52. On constate cependant que cette culture s'érode progressivement, particulièrement chez le Parti conservateur, qui s'aligne beaucoup plus sur les politiques pénales étasuniennes. 
système pénal a conservé de robustes théories et pratiques de l'Étatprovidence, et les professionnels du social ont constitué des remparts efficaces contre les excès du 'tournant punitif'» (p. 323). Plusieurs documents du Service correctionnel du Canada mettent en garde contre la voie suivie aux États-Unis et prônent le traitement des délinquants et la réinsertion sociale ${ }^{53}$. Il en est de même aux Services correctionnels du Québec ${ }^{54}$. Un bon nombre de cadres supérieurs et de professionnels de ces systèmes partagent cette idéologie et ils orientent leurs pratiques en conséquence $e^{55}$. On a aussi vu plusieurs propositions de politiciens ou même des projets de loi faire l'objet d'analyses critiques de la part de chercheurs ou d'experts à l'intérieur des ministères concernés.

\section{Conclusion}

«Le futur n'est pas inévitable.»

Les politiques pénales ont été grandement influencées par les politiques sociales conservatrices des dernières décennies. Les politiques de sécurité ont participé à une «culture de contrôle ${ }^{56}$ ». Devant de telles orientations, certains pourraient croire que nous sommes à la merci de forces structurelles et politiques irréversibles et ils pourraient se décourager ou, encore pire, accepter d'épouser ces orientations perçues comme inéluctables.

Mais «le futur n'est pas inévitable», comme le soulignait David Garland (2001). Ces orientations sont le résultat d'institutions politiques et de choix sociaux. Ces politiques et ces pratiques sont influencées par des forces structurelles profondes, mais elles sont néanmoins les conséquences de décisions prises par les décideurs politiques et aussi par les acteurs sociaux et en particulier ceux qui œuvrent dans le champ de la justice pénale ${ }^{57}$. Ces orientations peuvent être repensées et renversées. On peut remettre l'accent sur des politiques d'inclusion et de solidarité et favoriser de nouveau le principe de modération et une philosophie de réinsertion sociale ${ }^{58}$ en droit pénal. On voit d'ailleurs

53. Par exemple, Correctional Service of Canada (2001).

54. Par exemple, Lalande (2006).

55. Voir Moore et Hannah-Moffat (2005: 89).

56. Selon l'expression de Garland (2001).

57. Voir, entre autres, à ce sujet Snacken, Beyens et Tubex (1995).

58. La «Philosophie d'intervention en matière de réinsertion sociale aux Services correctionnels du Québec», adoptée en 2007 par les Services correctionnels du Québec à 
ressurgir plusieurs mouvements sociaux et politiques qui favorisent la solidarité, qui luttent contre les inégalités sociales et économiques et préconisent le refus de l'homogénéisation et de l'asservissement aux lois du marché.

Par ailleurs, comme le souligne O'Malley (2006): «Il ne faut pas voir dans les politiques pénales le reflet de courants punitifs monolithiques [...].» (p. 336). Au Canada, nous l'avons déjà mentionné, plusieurs agents sociaux œuvrant au sein des agences de l'administration de la justice pénale partagent les valeurs de modération en droit pénal, prônent le recours aux solutions de rechange à l'incarcération et résistent à leur niveau au mouvement répressif. Cette diversité d'orientations au sein même des appareils d'État explique en partie l'écart observé entre les discours punitifs et les pratiques. Même aux États-Unis, certains conservateurs remettent en cause "l'incarcération de masse ${ }^{59} »$. Des pratiques qui sont encore minoritaires ou à la marge, comme la justice réparatrice, peuvent permettre un questionnement des politiques et des pratiques répressives. Et enfin, le mouvement en faveur des droits de l'homme prend de plus en plus d'ampleur et cette question est de plus en plus soulevée par les tribunaux nationaux et supranationaux.

De plus, comme le propose Garland (2001), l'augmentation parfois considérable des infractions enregistrées par la police des années 1960 à 1990 a entraîné, chez les citoyens, particulièrement ceux des classes moyennes, une «nouvelle expérience de la criminalité» (p. 147) qui a miné le soutien pour des politiques pénales de tolérance et d'inclusion et favorisé l'émergence de pratiques punitives et de neutralisation. Si cette hypothèse est juste, la chute considérable des infractions enregistrées par la police au Canada pourrait laisser espérer une possibilité d'inversion des politiques répressives.

Nous avons vu que les acteurs sociaux et en particulier ceux qui œuvrent dans le champ de la justice pénale peuvent jouer un rôle important dans l'orientation des politiques et des pratiques pénales. Les criminologues, chercheurs, intervenants et décideurs, œuvrent dans ce que Nils Christie (1981) nomme une "pain delivery business». Ils participent à l'infliction délibérée de la souffrance à certains de leurs concitoyens. Il est donc normal qu'ils s'interrogent régulièrement sur leur rôle dans cette entreprise.

la suite de la mise en vigueur de la Loi sur le système correctionnel du Québec est encourageante à cet égard.

59. Garland (2001: 204). 
Le travail dans un système dont la raison d'être est de faire souffrir ses semblables, ou plutôt des êtres que l'on perçoit comme différents, mauvais ou dangereux, comporte l'énorme danger de s'habituer à la souffrance et de concevoir que ce que nous faisons est normal et même souhaitable. Nous devons donc être des plus vigilants et adopter une des prémisses morales proposées par Nils Christie, «that it is right to strive for a reduction of man-inflicted pain on earth» (1981: 10).

\section{Références}

Archambault, O. J. R. (Prés.). (1987). Réformer la sentence. Une approche canadienne. Rapport de la Commission canadienne sur la détermination de la peine. Ottawa: Ministre des Approvisionnements et services Canada.

Artières, Ph., \& Lascoumes, P. (Dir.). (2004). Gouverner et enfermer - La prison un modèle indépassable? Paris: Presses de Sciences-Po.

Boisvert, A-M., \& Dumont, H. (2004). Chronique Législative canadienne. Revue de science criminelle et de droit pénal comparé, 1, 203-209.

Bottoms, A. E. (1977). Reflexions on the Renaissance of Dangerousness. The Howard Journal of Penology and Crime Prevention, XVI (2), 70-95.

Brodeur, J.-P. (1999). Sentencing Reform: Ten Years after the Canadian Sentencing Commission. In J. Roberts (Ed.), Making Sense of Sentencing. Toronto: University of Toronto Press.

Canada (1984). La détermination de la peine. Ottawa: Ministère de la Justice.

Canada (1998). Pour une société juste, paisible et sûre. Rapport final. La loi sur le système correctionnel et la mise en liberté sous condition, cinq ans plus tard. Rapport du groupe de travail sur la LSCMLC. Ottawa: Ministre des travaux publics et Services gouvernementaux Canada.

Christie, N. (1981). Limits to Pain. Oslo: Columbia University Press.

Crutcher, N. (2001). Mandatory Minimum penalties of Imprisonment: An Historical Analysis. Criminal Law Quarterly, 44, 279-308.

Daubney, D. (Prés.). (1988). Des responsabilités à assumer. Rapport du Comité permanent de la justice et du Solliciteur général sur la détermination de la peine, la mise en liberté sous condition et d'autres aspects du système correctionnel. Chambre des Communes, fascicule no 65. Ottawa: Ministère des Approvisionnements et services Canada.

Dumont, H. (1997). Désarmons les Canadiens et armons-nous de tolérance. Revue canadienne de droit pénal, 2, 43-70.

Faugeron, C., \& Le Boulaire, J. M. (1992). Prisons, peines de prison et ordre public. Revue française de sociologie, 33 (1), 3-32.

Fauteux, G. (Prés.). (1956). Rapport d'un comité institué pour faire enquête sur les principes et les méthodes suivis au service des pardons du ministère de la Justice $d u$ Canada. Ottawa: Imprimeur de la Reine et contrôleur de la papeterie. 
Feeley, M., \& Simon, J. (1992). The New Penology: Notes on the Emerging Strategy or Corrections and its Implication. Criminology, 30 (4), 449-474.

Gannon, M. (2006). Statistiques de la criminalité au Canada, 2003. Juristat, 26, $\mathrm{n}^{\circ}$ 4, Ottawa: Statistique Canada.

Garland, D. (1995). Penal Modernism and Postmodernism. In T. G. Blomberg $\&$ S. Cohen (Eds.), Punishment and Social Control (181-210). New York: Aldine de Gruyer.

Garland, D. (2001). The Culture of Control, Crime and Social Order in Contemporary Society. Oxford: Oxford University Press.

Gaudreault, A. (2001a). Évolution de la législation et des politiques concernant les besoins des victimes d'actes criminels dans le système correctionnel du Canada. In E. Fattah \& S. Parmentier (Eds.), Victim policies and Criminal Justice on the Road to Restorative justice (99-111). Leuven: Leuven Press University.

Gaudreault, A. (2001b). L'aide aux victimes d'actes criminels au Canada et au Québec: esquisse d'un parcours. In R. Cario \& D. Salas (Dir.), Euvre de justice et victimes (109-122). Paris: L'Harmattan.

Groupe d'étude fédéral-provincial canadien sur la justice pour les victimes d'actes criminels (1983). La justice pour les victimes d'actes criminels. Ottawa: Ministre des Approvisionnements et services.

Hatt, K., Caputo, T., \& Perry, B. (1992). Criminal Justice Policy Under Mulroney, 1984-90: Neo-Conservatism, Eh? Canadian Public Policy, XVIII (3), 245-260.

Laberge, D., \& Landreville, P. (1991). La justice pénale au Québec, 1960-1990. Recherches sociographiques, XXXII (2), 199-220.

Lalande, P. (2006). Punir ou réhabiliter les contrevenants? Du «Nothing Works» au «What Works». In Ministère de la Sécurité publique, La sévérité pénale à l'heure du populisme. Québec: Ministère de la Sécurité publique.

Landreville, P. (1995). Prison Overpopulation and Strategies for Decarceration. Revue canadienne de criminologie, 37 (1), 39-60.

Landreville, P. (2001). Le vieillissement de la population pénitentiaire du Québec. Sociologie et sociétés, 33 (1), 53-66.

Landreville, P. (2002). Va-t-on vers une américanisation des politiques pénales en Europe? In L. Mucchielli \& Ph. Robert (Dir.), Crime et sécurité. L'état des savoirs. Paris: Éditions La Découverte.

Landreville, P., Gagnon, A., \& Desrosiers, S. (1976). Les prisons de par ici. Montréal: Éditions Parti pris.

Landreville, P., Laberge, D., Morin, D., \& Casavant, L. (1998). Logique d'action et fonctions de la prison. L'exclusion des itinérants par le droit pénal. In $\mathrm{Ph}$. Robert (Dir.), La gestion sociale par le droit pénal. La discipline du travail et la punition des pauvres (153-170). Cowansville: Les Éditions Yvon Blais Inc.

Landreville, P., \& Charest, M. (2004). Analyse prospective des établissements de détention du Québec, rapport final. Montréal: Centre international de criminologie comparée. 
Lascoumes, P. (2006). Ruptures politiques et politiques pénitentiaires, analyse comparative des dynamiques de changement institutionnel. Déviance et Société, 30 (3), 405-419.

Lemonde, L., \& Landreville, P. (2002). La reconnaissance des droits fondamentaux des personnes incarcérées: l'expérience canadienne. In D. Kaminski \& O. DeSchutte (Dir.), L'institution du droit pénitentiaire. Enjeux de la reconnaissance des droits aux détenus (69-88). Paris: Bruylant, L. G. D. J.

Martin, M. (Prés.). (1954). Rapport de la Commission royale pour la révision du code criminel. Ottawa: Imprimeur de la Reine et contrôleur de la papeterie.

Mary, Ph. (2001). Pénalité et gestion des risques: vers une justice «actuarielle» en Europe. Déviance et société, 25 (1), 33-52.

Meyer, J., \& O’Malley, P. (2005). Missing the Punitive Turn? Canadian Criminal Justice, «Balance» and Penal Modernism. In J. Pratt, D. Brown, M. Brown, S. Hallsworth, \& W. Morrison (Eds.), The New Punitiveness. Trends, Theories, Perspectives (201-217). Portland: William Publishing.

Moore, D., \& Hannah-Moffat, K. (2005). The Liberal Veil: Revisiting Canadian Penalty. In J. Pratt, D. Brown, M. Brown, S. Hallsworth, \& W. Morrison (Eds.), The New Punitiveness. Trends, Theories, Perspectives (85-100). Portland: William Publishing.

O'Malley, P. (1999). Volatile and Contradictory Punishment. Theoretical Criminology, 3, 175-196.

O'Malley, P. (2006). «Mondialisation» et justice criminelle: du défaitisme à l'optimisme. Déviance et Société, 30 (3), 323-338.

Ouimet, M. (2002). Explaining the American and Canadian Crime «Drop» in the 1990's. Revue canadienne de criminologie, 44 (1), 33-50.

Ouimet, M. (2004). Oh Canada! La baisse de la criminalité au Canada et aux États-Unis entre 1991 et 2002. Champ pénal, http://champpenal.revues. org

Ouimet, M. (Prés.). (1969). Rapport du Comité canadien de la réforme pénale et correctionnelle. Ottawa: Imprimeur de la Reine.

Pires, A. P. (1998). Aspects, traces et parcours de la rationalité pénale moderne. In C. Debuyst, F. Digneffe \& A. P. Pires (Dir.), Les savoirs sur le crime et la peine. La rationalité pénale et la naissance de la criminologie (vol. 2, 3-52). Bruxelles: De Boeck Université.

Pratt, J., Brown, D., Brown, M., Hallsworth, S., \& Morrison, W. (2005). (Eds.). The New Punitiveness. Trends, Theories, Perspectives. Portland: William Publishing.

Prévost, Y. (Prés.). (1968). La société face au crime. Commission d'enquête sur l'administration de la justice en matière criminelle et pénale au Québec. Québec: Lefebvre (5 vols.).

Robert, D. (2001). Transformations récentes de la législation fédérale sur la mise en liberté sous condition au Canada. Criminologie, 34 (1), 73-99.

Roberts, J., \& Doob, A. (1997). Race, Ethnicity, and Criminal Justice in Canada. In M. Tonry (Ed.), Ethnicity, Crime and Immigration. Comparative and Cross- 
National Perspectives. Crime and Justice, 21. Chicago: The University of Chicago Press.

Roberts, J., Stalans, L. J., Indermaur, D., \& Hough, M. (2003). Penal Populism and Public Opinion, Lessons from Five countries. Oxford: Oxford University Press.

Sabol, W. J., Minton, T. D., \& Harrison, P. M. (2007). Prison and Jail Inmates at Midyear 2006. Bureau of Justice Statistics Bulletin, www.ojp.usdoj.gov/ bjs/

Snacken, S., Beyens, K., \& Tubex, H. (1995). Changing prison populations in western countries: fate or policy? European Journal of Crime, Criminal Law and Criminal Justice, 1, 18-53.

Tonry, M. (2005). Malign Neglect: Race, Crime and Punishment in America. New York: Oxford University Press.

Trépanier, J. (1986). La justice des mineurs au Québec: 25 ans de transformation, 1960-1985. Criminologie, XIX (1), 189-213.

Turner, J. (1993). La détermination de la peine dans les tribunaux provinciaux de juridiction criminelle pour adultes au Canada. Ottawa: Statistique Canada.

Vacheret, M., Lemire, G., \& Dozois, J.(1998). Le système correctionnel canadien et la nouvelle pénologie: la notion de risque. Déviance et société, 22 (1), 3750 .

Wacquant, L. (1999). Les prisons de la misère. Paris: Raisons d'agir.

Wacquant, L. (2005). The Great Penal Leap Backward: Incarceration in America from Nixon to Clinton. In J. Pratt, D. Brown, M. Brown, S. Hallsworth \& W. Morrison (Eds.), The New Punitiveness. Trends, Theories, Perspectives (85100). Portland: William Publishing.

Zedner, L. (2002). Dangers of Dystopia in Penal Theory. Oxford Journal of Legal Studies, 22, 341-366. 